\title{
Cationic metal-organic framework based mixed-matrix membrane for extraction of phenoxy carboxylic acid (PCA) herbicides from water samples followed by UHPLC-MS/MS determination
}

\author{
Gege $\mathrm{Wu}^{\mathrm{a}}$, Jiping $\mathrm{Ma}^{\mathrm{a}, * *}$, Shasha Wang ${ }^{\mathrm{a}}$, Huining Chai ${ }^{\mathrm{a}}$, Liang $\mathrm{Guo}^{\mathrm{c}}$, Jinhua $\mathrm{Li}^{\mathrm{b}}$, Abbas Ostovan ${ }^{\mathrm{b}}$, \\ Yafeng Guan ${ }^{\mathrm{d}}$, Lingxin Chen ${ }^{\mathrm{b}, \mathrm{e}, *}$ \\ ${ }^{\text {a }}$ School of Environmental \& Municipal Engineering, Qingdao University of Technology, Qingdao, 266033, China \\ ${ }^{\mathrm{b}}$ CAS Key Laboratory of Coastal Environmental Processes and Ecological Remediation, Research Center for Coastal Environmental Engineering and Technology, Yantai \\ Institute of Coastal Zone Research, Chinese Academy of Sciences, Yantai, 264003, China \\ ${ }^{\mathrm{c}}$ Qingdao Junray Intelligent Instrument Co., Ltd., Qingdao, 266000, China \\ d CAS Key Laboratory of Separation Sciences for Analytical Chemistry, Dalian Institute of Chemical Physics, Chinese Academy of Sciences, Dalian, 116023, China \\ ${ }^{\mathrm{e}}$ Center for Ocean Mega-Science, Chinese Academy of Sciences, Qingdao, 266071, China
}

\section{G R A P H I C A L A B S T R A C T}

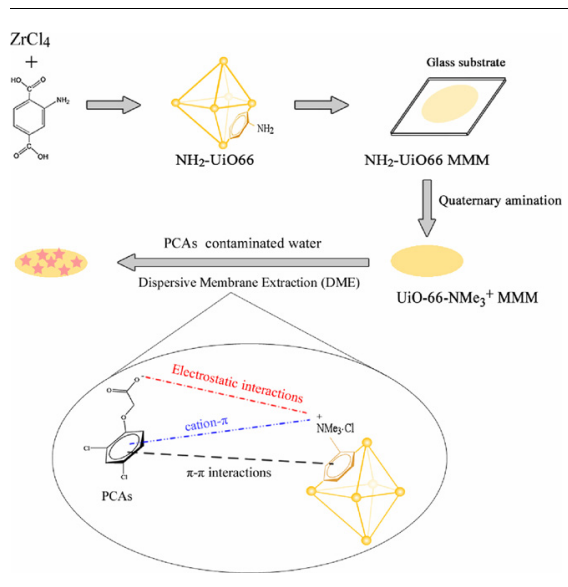

\section{A R T I C L E I N F O}

Editor: Navid B. Saleh

Keywords:

Ionic metal-organic frameworks (MOFs)

Mixed-matrix membrane (MMM)

Dispersion membrane extraction (DME)

Phenoxy carboxylic acid (PCA) herbicides

Water sample

\begin{abstract}
A B S T R A C T
A novel kind of cationic metal-organic framework(MOF) based mixed-matrix membrane(MMM) namely cationic MOF-MMM was firstly designed and used for simultaneous dispersive membrane extraction(DME) of six phenoxy carboxylic acid(PCA) herbicides from water samples followed by determination using ultrahigh-performance liquid chromatography tandem mass spectrometry. The cationic MOF-MMM was synthesized by soaking the zirconium-based MOFs in a polyvinylidene fluoride(PVDF) solution and further functionalization with quaternary amine groups, viz., UiO-66- $\mathrm{NMe}_{3}{ }^{+} \mathrm{MMM}$. The well-prepared UiO-66- $\mathrm{NMe}_{3}{ }^{+} \mathrm{MMM}$ was characterized by FT-IR, SEM, XRD, XPS, NMR and etc. Several main variables influencing the MMM based DME efficiency were investigated and optimized in detail, such as dosage ratio of MOF/PVDF, solution $\mathrm{pH}$, extraction time, coexistent
\end{abstract}

\footnotetext{
* Corresponding author at: CAS Key Laboratory of Coastal Environmental Processes and Ecological Remediation, Research Center for Coastal Environmental Engineering and Technology, Yantai Institute of Coastal Zone Research, Chinese Academy of Sciences, Yantai, 264003, China.

** Corresponding author at: School of Environmental \& Municipal Engineering, Qingdao University of Technology, Qingdao, 266033, China.

E-mail addresses: majiping2012@163.com (J. Ma), lxchen@yic.ac.cn (L. Chen).
} 
anions and ionic strength. Electrostatic interactions dominated adsorption mechanism between anionic PCAs and cationic UiO-66- $\mathrm{NMe}_{3}{ }^{+} \mathrm{MMM}$, along with $\pi-\pi$ conjugation and cation- $\pi$ bonding, leading to better adsorption performance. Low limits of detection in the range of $0.03-0.59 \mathrm{ng} / \mathrm{L}$ and satisfactory recoveries within 80.06-117.40 \% for all the PCAs are a reliable witness to demonstrate supreme sensitivity and the applicability of the developed method. By relying on the obtained results, the present work implied cationic MOF-MMM based DME can be a versatile and worthy utility for extraction of pollutants from different water samples with high throughput.

\section{Introduction}

To increase the production of more quality agricultural crops, annually too many tons of chemical fertilizers, herbicides, insecticides, and etc. are consumed. Due to the excellent efficiency and low cost, phenoxy carboxylic acid (PCA) herbicides have been widely used as plant growth regulator. However, PCAs are high toxicity for human's kidney, liver and central nervous system and also cause the endocrine disorder. One of these herbicides namely 2,4-dichlorophenoxybutyric acid $(2,4-\mathrm{D})$ has been classified as a potential human carcinogen and mutagen by the International Agency for Research on Cancer (Arcaute et al., 2016). The maximum residue limits (MRLs) of PCAs in drinking water set by the World Health Organization are in the range of $2-100 \mu \mathrm{g} / \mathrm{L}$ (Ghambarian et al., 2017). Excessive residues of PCAs can easily leak into natural environment water source through natural drainage or infiltration, leading to a serious threat to public health. Thus, it is of great significance to establish sensitive and simple analytical methods for monitoring and quantitative analysis of residual PCAs in the environmental water samples at trace level. Various analytical technologies, including gas chromatography mass spectrometry (GC-MS) (Suárez et al., 2018), high-performance liquid chromatography (HPLC) (Wu et al., 2005; Mojtaba et al., 2012), liquid chromatography tandem mass spectrometry (LC-MS/MS) (Peng et al., 2018; Ma et al., 2019b; Gao et al., 2014; Jia et al., 2019) and capillary electrophoresis (CE) (Fu et al., 2009), have been used for detecting the amounts of PCAs. Amongst them, LC-MS/MS exhibits the highest sensitivity and accuracy, which is more suitable for the PCAs analysis. However, PCAs at ultra-low concentrations in the real samples need to be enriched along with elimination of matrix interferences prior to instrumental analysis by efficient pretreatment technique.

Among available sample preparation technologies, solid-phase extraction (SPE) is especially favorable for concurrent enrichment and clean-up of various compounds from complicated matrices. Owing to the aim of the analysis and the state of the sample, SPE can be used in different forms like packed cartridge, dispersive or membrane mode. The membrane SPE mode benefits from high mass transfer efficiency and ease of operation for solid-liquid separation. It is well known that the selectivity and efficiency of SPE are largely dependent on the characteristics of solid adsorbent materials. For membrane or disk SPE mode, $\mathrm{C}_{18}$ membrane is the mostly used commercial membrane adsorbent material. However, $\mathrm{C}_{18}$ membrane does not possess satisfactory selectivity toward some analytes. Nowadays, a wide variety of new synthetic adsorbents are available and have been utilized in the membrane extraction, such as molecularly imprinted polymers (Mhaka et al., 2009), multi-walled carbon nanotubes (Sanagi et al., 2015), and metal-organic frameworks (MOFs) (Gao et al., 2018). MOFs are a class of porous materials with metal node or clusters interconnected by organic linkers. MOFs currently have received considerable attention due to their unique properties, such as large specific surface area, controlled porosity, and well-defined adsorption sites.

Owing to these prominent superiorities, MOFs have been variously applied in the fields of gas storage (Xue et al., 2019), catalysis (Chen and Wu, 2019), drug delivery (Wu and Yang, 2017), sensing (Zhu et al., 2013) and especially in the sample preparation (Mondal et al., 2019; Lin et al., 2015). Our group has prepared magnetic MIL-101 and magnetic MOF-5 and utilized them in magnetic SPE for extraction of pyrazole/pyrrole pesticides (Ma et al., 2016) and heterocyclic pesticides (Ma et al., 2018), respectively. Both MIL-101 and MOF-5 can capture target compounds containing a benzene ring through $\pi-\pi$ interactions. However, the bond force of $\pi-\pi$ interactions is week and the selectivity was not desirable. On the other hand, after extraction process, separation of MOFs from solution needs centrifugation or applying an external magnetic field for magnetic MOFs. Indeed, by the both of mentioned approaches, MOFs cannot be separated completely and a few amounts of them are remained in the solution and cause subsequent troubles. To overcome the noted problem, we have incorporated MIL53 to the membrane and designed streamlined approach for extraction of sulfonylurea herbicides in water (Ma et al., 2019a). Howbeit, poor affinity and specificity of MOF mixed membranes toward target species is still faced.

Up to now, most of the reported MOFs are electrically neutral, since the positive charges of the metal ion in MOFs are exactly coordinated with electronegative organic ligands like carboxylate-based linkers (Farha et al., 2007; Xiang et al., 2010). Thus, altering the electronic properties of MOFs by functionalization process is promising to extend their applicability as adsorbents toward polar substances. Ionic MOFs (iMOFs) are a new class of MOFs that contain residual charge within their framework, which can exhibit excellent ion-exchange/sorption properties (Rapti et al., 2016; Howarth et al., 2015). Several reported iMOFs have demonstrated excellent selectivity toward targeted ionic compounds. Liu et al. (2015) have used preassembled modification (PAM) and post synthesis modification (PSM) method to synthesize anionic-exchange MIL-101(Cr), and subsequently applied it for the adsorption removal of perfluorooctanoic acid from aqueous solution. Zhang et al. (2017) have functionalized MIL-101(Cr) with quaternary amine groups and combined it with $\mathrm{Fe}_{3} \mathrm{O}_{4} @ \mathrm{SiO}_{2}$ under ultrasound waves. The magnetic iMOFs have been used for extraction of azide in sartan drugs. These kinds of researches have proved that iMOFs material can remarkably promote the adsorption performance and selectivity of SPE approaches compared with pristine MOFs based SPE.

Therefore, herein, we introduce a facile procedure for fabrication of new iMOF mixed-matrix membrane (MMM) namely UiO-66- $\mathrm{NMe}_{3}{ }^{+}$ MMM for simultaneous dispersive membrane extraction (DME) (a dispersive SPE mode) of six PCAs followed by HPLC-MS/MS determination. UiO-66- $\mathrm{NH}_{2}$ was employed as the pristine MOFs and anchored into the MMM, further functionalized with the quaternary amine group to fabricate a novel cationic MOF MMM. The resultant UiO-66- $\mathrm{NMe}_{3}{ }^{+}$ MMM was thoroughly characterized, the MMM based DME conditions were systematically optimized, and possible adsorptive extraction mechanism of PCAs by MMM was proposed. The developed DME-HPLCMS/MS method was well validated and practically applied. To the best of our knowledge, this is the first report about the synthesis and application of UiO-66- $\mathrm{NMe}_{3}{ }^{+} \mathrm{MMM}$ for the extraction of trace PCAs in water samples.

\section{Experimental}

\subsection{Reagents and materials}

Six PCA herbicides including 2-methyl-4-chlorophenoxyacetic acid (MCPA), 2,4-dichlorophenoxyacetic acid (2,4-D), dichlorprop (2,4-DP) and 2,4-dichlorophenoxybutyric acid (2,4-DB) were purchased from 
Shanghai Aladdin Chemistry Co., Ltd. (Shanghai, China), and 2,4,5trichlorophenoxyacetic acid (2,4,5-T) and 2-(2,4,5-trichlorophenoxy) propanoic acid (2,4,5-TP) were obtained from Dr. Ehrenstorfer GmbH (Germany). The structural formula of the six PCAs and their $\mathrm{p} K_{\mathrm{a}}$ values were shown in Fig. S1. Stock standard solutions of the PCAs at $1.0 \mathrm{mg} /$ $\mathrm{mL}$ were prepared in methanol and stored at $4{ }^{\circ} \mathrm{C}$. Zirconium chloride $\left(\mathrm{ZrCl}_{4}\right)$ was acquired from Sinopharm Chemical Reagent Co. Ltd. (Shanghai, China). 2-Amino-terephthalic acid and methyl triflate were purchased from Shanghai Aladdin Chemistry Co., Ltd. (Shanghai, China). Poly (vinylidene fluoride) (PVDF) was purchased from American Arkema Company (USA). Dichloromethane, ethanol and dimethylformamide (DMF) were supplied from Aibi Chemical Reagent Limited Company (Shanghai, China). HPLC grade methanol and acetonitrile were obtained from Merck (Germany). Ultrapure water (18 M $\Omega$ resistance) was obtained from a MingcheD-24UV purification device (Millipore, France).

Water samples were collected from LiCun sewage treatment plant (Qingdao, China) and the NuoCheng reservoir (Qingdao, China). All samples were filtered and reserved in brown glass bottles at $4{ }^{\circ} \mathrm{C}$ before analysis.

\subsection{Instruments for characterization}

Frontier Nicolet iN10 infrared spectrometer (Thermo Fisher, USA) with an attenuated total reflection (ATR) accessory in the wave number range from 4000 to $400 \mathrm{~cm}^{-1}$ was used to identify the functional groups of materials. D8 Advance (Bruker, USA) was used to record the $\mathrm{X}$-ray diffractometer (XRD) patterns. Shape and morphology of UiO-66$\mathrm{NMe}_{3}{ }^{+}$MMM membrane was investigated by SUPRA 55 scanning electron microscope (SEM, ZEISS, Germany). Surface chemical composition of the materials was study by Thermo SCIENTIFIC ESCALAB 250Xi (Thermo Fisher, USA) X-Ray photoelectron spectroscopy (XPS). Bruker AVANCE III $600 \mathrm{~Hz}$ (Bruker, USA) was used to prove successful functionalization of materials by record nuclear magnetic resonance (NMR). CMT6500 tensiometer (XinSanxi, China) was employed to measure the mechanical strength of UiO-66- $\mathrm{NMe}_{3}{ }^{+} \mathrm{MMM}$ at room temperature with humidity of $65 \%$. Pore size of the membrane was estimated via capillary flow porometer (Porometer 3G, USA) with bubble point measurement. The hydrophilicity of MOFs was tested by DCAT11 tensiometer (Dataphysics, Germany) for the water contact angle. $\mathrm{N}_{2}$ adsorption-desorption experiment was carried out on a $3 \mathrm{H}$ -
2000PS1 Instrument (BeiShiDe, China) for Brunauer-Emmett-Teller (BET) analysis to determine the specific surface area and pore size.

\subsection{UHPLC-MS/MS conditions}

PCAs determination was performed on a SCIEXExion LC coupled with SCIEX QTRAP $3500 \mathrm{MS}$ /MS system (SCIEX, USA) equipped with electrospray ionization (ESI) source. Phenomenex Kinetex F5 column $(50 \times 3.0 \mathrm{~mm}, 2.6 \mu \mathrm{m})$ was employed for UHPLC separation at $40^{\circ} \mathrm{C}$. Eluent A was ultrapure water containing $0.1 \%$ formic acid and B was pure acetonitrile. The gradient condition was as follows: $97 \% \mathrm{~A}$ kept from 0 to $1 \mathrm{~min}, 95-25 \%$ A from 1.1 to $9.5 \mathrm{~min}, 5 \% \mathrm{~A}$ kept from 9.6 to $11.5 \mathrm{~min}, 97 \% \mathrm{~A}$ kept from 11.6 to $13.5 \mathrm{~min}$. The flow rate was set at $0.4 \mathrm{~mL} / \mathrm{min}$ and the sample injection volume was $5 \mu \mathrm{L}$. The MS analysis was operated at the negative ionization mode. The ESI source parameters were as follows: $-4500 \mathrm{~V}, 30 \mathrm{psi}, 50 \mathrm{psi}, 60 \mathrm{psi}, 600^{\circ} \mathrm{C}$, for ion source voltage, curtain gas, nebulizing gas, auxiliary gas and ion source temperature, respectively. The optimal UHPLC-MS/MS conditions with the most intense MS transitions were shown in Table S1.

\subsection{Preparation of UiO-66- $\mathrm{NMe}_{3}{ }^{+} \mathrm{MMM}$}

First, UiO-66- $\mathrm{NH}_{2}$ was synthesized by a typical solvothermal method (Massoudinejad et al., 2016). Briefly, $\mathrm{ZrCl}_{4}(0.23 \mathrm{~g}$ ) and 2amino-terephthalic acid $(0.16 \mathrm{~g})$ were dissolved in $50 \mathrm{~mL}$ DMF. The mixture was transferred to a Teflon-lined autoclave, sealed and maintained at $120^{\circ} \mathrm{C}$ for $48 \mathrm{~h}$. After cooling naturally to room temperature, the obtained powder was isolated by centrifugation and washed with DMF. Subsequently, UiO-66- $\mathrm{NH}_{2}$ was further purified by washing with ethanol to remove the unreacted substances. The resulting UiO-66- $\mathrm{NH}_{2}$ was dried at $70{ }^{\circ} \mathrm{C}$ for $12 \mathrm{~h}$. Afterwards, $60 \mathrm{mg}$ of UiO-66- $\mathrm{NH}_{2}$ powder was dispersed thoroughly in $2 \mathrm{~mL}$ acetone by sonication. $1 \mathrm{~mL}$ of PVDF solution ( $5 \mathrm{wt} \%$ in DMF) was added to the UiO-66- $\mathrm{NH}_{2}$ suspension. The UiO-66- $\mathrm{NH}_{2}$ /PVDF suspension was further mixed under ultrasound for $15 \mathrm{~min}$, followed by transferring to rotary evaporation to remove acetone. The obtained mixture of UiO-66- $\mathrm{NH}_{2}$ and PVDF in DMF was uniformly poured onto a clean glass plate. The coated glass substrate was then heated at $70{ }^{\circ} \mathrm{C}$, and UiO-66- $\mathrm{NH}_{2}$ membrane was gradually formed when the solvent was dried. The membrane was delaminated from glass substrate by immersing in methanol, and the freestanding UiO-66- $\mathrm{NH}_{2}$ membrane was dried in air.
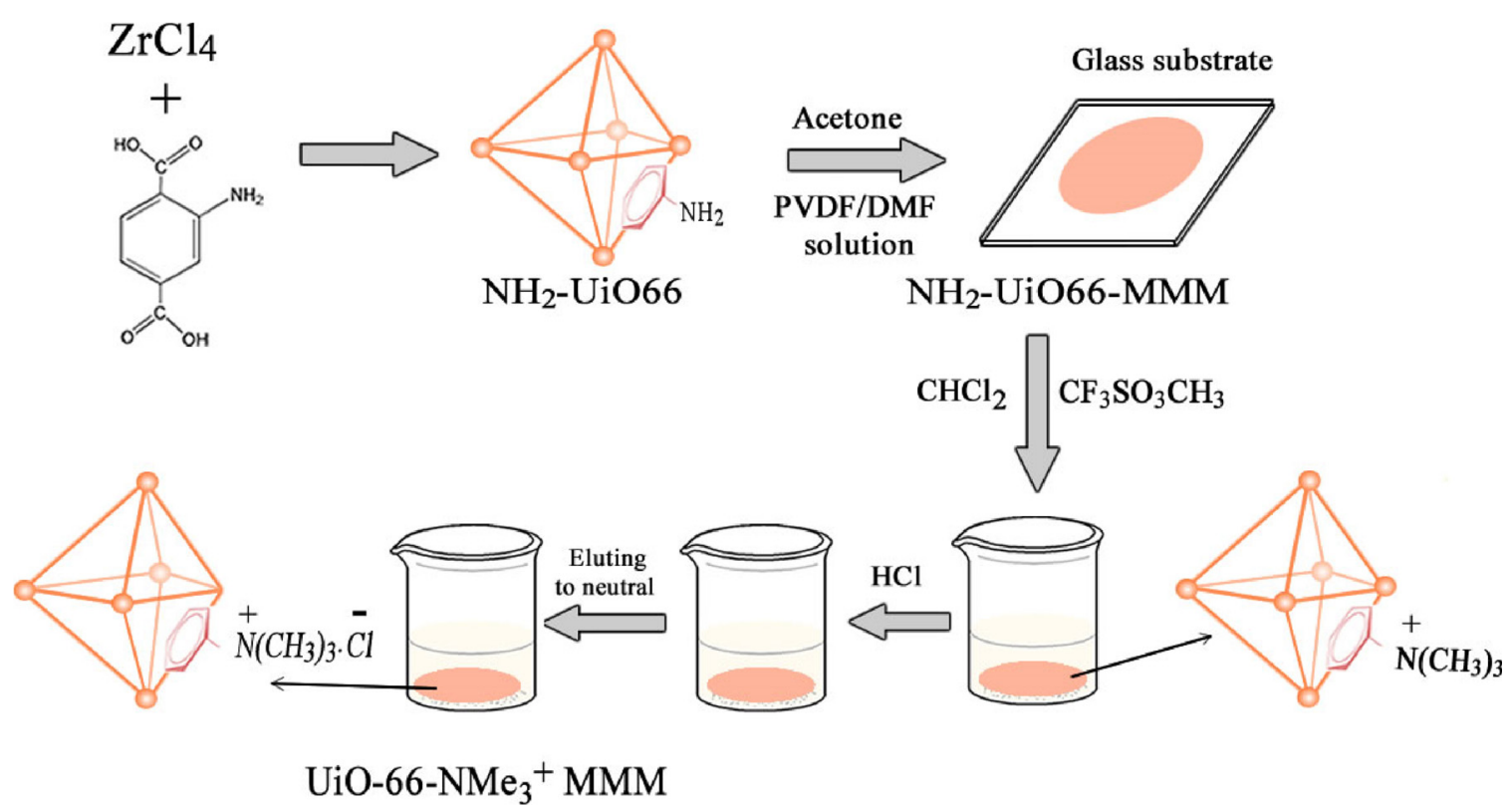

Fig. 1. Illustration for the synthesis of the UiO-66- $\mathrm{NMe}_{3}{ }^{+} \mathrm{MMM}$. 
The prepared UiO-66- $\mathrm{NH}_{2}$ membrane was immersed in $6 \mathrm{~mL}$ dichloromethane and $70 \mu \mathrm{L}$ methyl triflate was dropped in the mixture. The quarter of amination reaction was conducted during $12 \mathrm{~h}$ at room temperature. The membrane was soaked in dichloromethane and the solvent was replaced every $12 \mathrm{~h}$ for three times to remove unreacted methyl triflate, followed by drying in air. Finally, the resulting UiO-66$\mathrm{NMe}_{3}$ membrane was soaked in $0.1 \mathrm{M} \mathrm{HCl}$ for $12 \mathrm{~h}$ at room temperature. Afterwards, the acidified membrane was washed repeatedly with ultrapure water until the $\mathrm{pH}$ of eluent reaching neutral and cationic UiO-66- $\mathrm{NMe}_{3}{ }^{+}$MMM was obtained. The synthesis procedure was schematically shown in Fig. 1.

\subsection{UiO-66- $\mathrm{NMe}_{3}{ }^{+} \mathrm{MMM}$ based DME procedure}

The prepared UiO-66- $\mathrm{NMe}_{3}{ }^{+}$MMM was immersed in $50 \mathrm{~mL}$ spiked water sample $(150 \mathrm{ng} / \mathrm{L})$ in a conical flask, and the flask was placed in a shaker with constant temperature for $30 \mathrm{~min}$. After extraction, the membrane was removed and placed into a $100 \mathrm{~mL}$ beaker and washed twice with $5 \mathrm{~mL}(2.5 \times 2)$ of methanol containing $1 \%(\mathrm{v} / \mathrm{v})$ ammonia. The desorbed solution was combined and dried with a gentle stream of nitrogen at room temperature, dissolved in $0.5 \mathrm{~mL}$ pure water containing $10 \%(\mathrm{v} / \mathrm{v})$ methanol, and ready for UHPLC-MS/MS analysis. The extraction procedure was shown schematically in Fig. S2.

\section{Results and discussion}

\subsection{Choice of membrane material}

Robust coordination bonds and high degree of topological connectivity within UiO-66 frameworks afford them large surface area, high thermal stability, and especially great hydro stability (Jasmina et al., 2008; Wang et al., 2015). Thus, it was selected as the pristine MOF for further functionalization. As the previous research reported (Denny and Cohen, 2015), post synthesis modification is accessible for MOFs based MMM. Bearing these in mind, quaternary amine functionalization of UiO-66 MMM was implemented to fabricate cationic MOFs MMM. The new material possessed abundant benzene rings and positive charge frameworks, which were beneficial to form $\pi-\pi$ conjugation and electrostatic interactions simultaneously to trap anionic PCAs. On the other hand, by deposition of UiO-66 onto the membrane, dispersion and isolation of adsorbent was performed easily without needing centrifuge. Therefore, UiO-66- $\mathrm{NMe}_{3}{ }^{+}$MMM based DME pretreatment technique provided an appropriate approach to enrich trace PCAs from water samples.

\subsection{Characterization of the cationic membrane}

The well-prepared cationic membrane UiO-66- $\mathrm{NMe}_{3}{ }^{+} \mathrm{MMM}$ were fully characterized by SEM, FT-IR, XRD, XPS, NMR and so on. The morphology of initial UiO-66- $\mathrm{NH}_{2} \mathrm{MMM}$ and UiO-66- $\mathrm{NMe}_{3}{ }^{+} \mathrm{MMM}$ was characterized by SEM. As seen in Fig. 2(A and B), the UiO-66- $\mathrm{NH}_{2} \mathrm{MOF}$ was successfully synthesized with octahedral structure, and the MOF

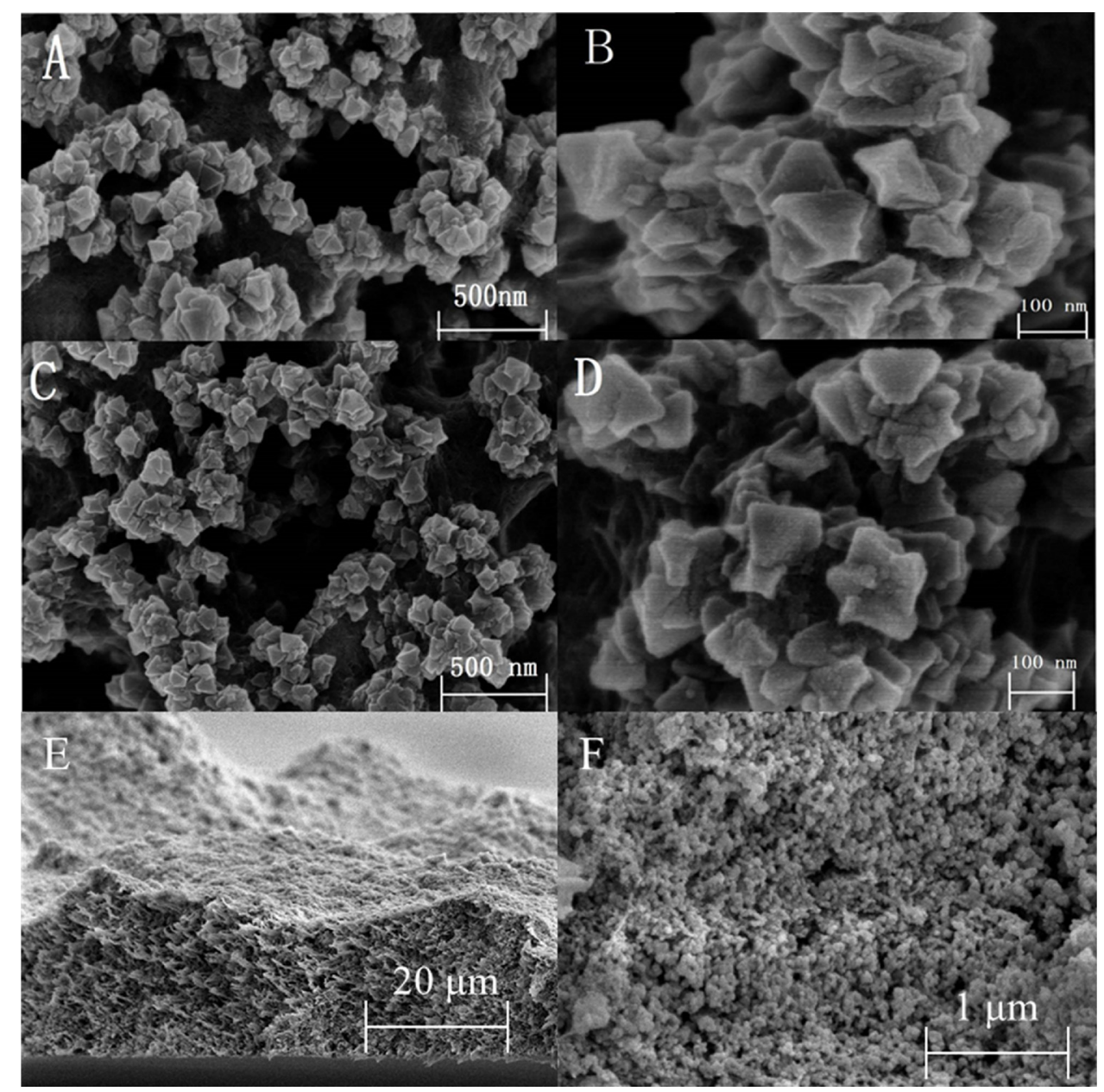

Fig. 2. SEM images of UiO-66- $\mathrm{NH}_{2}$ MMM with the scale bar of $500 \mathrm{~nm}$ (A) and $100 \mathrm{~nm}$ (B), UiO-66- $\mathrm{NMe}_{3}{ }^{+} \mathrm{MMM}$ with the scale bar of $500 \mathrm{~nm}$ (C) and $100 \mathrm{~nm}$ (D), and cross-sectional SEM images of UiO-66- $\mathrm{NMe}_{3}{ }^{+}$MMM with the scale bar of $20 \mu \mathrm{m}$ (E) and $1 \mu \mathrm{m}(\mathrm{F})$. 
microcrystals were cross-linked with each other by the polymer binder. The SEM images of UiO-66- $\mathrm{NH}_{2}$ MMM are consistent with that reported (Denny and Cohen, 2015). The UiO-66-NMe ${ }^{3+}$ MMM was characterized by cross-sectional SEM as well. Fig. 2E and F show the PVDF membrane had a sponge-like structure with an approximate thickness of $20 \mu \mathrm{m}$. The PVDF matrix provided ideal polymeric binder, which contained abundant active sites for cross-linking MOFs microcrystals (Denny and Cohen, 2015). Consequently, the excellent compatibility between UiO66- $\mathrm{NMe}_{3}{ }^{+}$and PVDF matrix ensured the facile fabrication of UiO-66$\mathrm{NMe}_{3}{ }^{+}$MMM. The morphology comparison of UiO-66- $\mathrm{NMe}_{3}{ }^{+} \mathrm{MMM}$ and UiO-66- $\mathrm{NH}_{2}$ MMM (Fig. 2A-D) revealed that the functionalization process didn't have obvious effect on the structure of UiO-66- $\mathrm{NH}_{2}$ MMM. Hence, other characterizations were carried out in order to confirm the successful functionalization.

The FT-IR spectra of PVDF (a), UiO-66- $\mathrm{NH}_{2}$ MMM (b) and UiO-66$\mathrm{NMe}_{3}{ }^{+}$MMM (c) are displayed in the Fig. 3. As shown in Fig. 3a, the absorption band at $1200 \mathrm{~cm}^{-1}$ could be related to C-F stretching vibration. Two absorption peaks at $1386 \mathrm{~cm}^{-1}$ and $1655 \mathrm{~cm}^{-1}$ existing in UiO-66- $\mathrm{NH}_{2}$ MMM spectra (Fig. 3b) could be attributed to $\mathrm{O}-\mathrm{C}-\mathrm{O}$ groups of 2-amino-terephthalic acid. The bands at $1496 \mathrm{~cm}^{-1}$ and $1571 \mathrm{~cm}^{-1}$ can be ascribed to $\mathrm{C}=\mathrm{C}$ stretching bands of the benzene ring. Furthermore, the band at $1254 \mathrm{~cm}^{-1}$ and $967 \mathrm{~cm}^{-1}$ could be assigned to the $\mathrm{C}-\mathrm{N}$ stretching vibration. These results demonstrated that the amino group of terephthalic acid ligand was incorporated in the framework of UiO-66- $\mathrm{NH}_{2}$ MMM. In the UiO-66- $\mathrm{NMe}_{3}{ }^{+}$MMM spectra (Fig. 3c), the $\mathrm{C}-\mathrm{N}$ stretching vibration band at $967 \mathrm{~cm}^{-1}$ shifted to $1072 \mathrm{~cm}^{-1}$, suggesting the formation of quaternary ammonium groups (Makvandi et al., 2016). Moreover, a new emerging vibration band at $1280 \mathrm{~cm}^{-1}$ corresponding to $\mathrm{C}-\mathrm{N}$ stretching also revealed the presence of quaternary ammonium groups.

Fig. 4 shows the XRD patterns of UiO-66- $\mathrm{NH}_{2}$ MMM (a) and UiO-66$\mathrm{NMe}_{3}{ }^{+}$MMM (b), and the simulated XRD pattern of UiO-66 crystal (c). As seen, the diffraction peaks at $2 \theta=7.4,8.6$ and $25.8^{\circ}$ can be observed in UiO-66- $\mathrm{NH}_{2}$ MMM (a) and functionalized UiO-66- $\mathrm{NMe}_{3}{ }^{+}$ MMM (b), which are matching with the simulated XRD pattern of initial UiO-66 crystal (c), as well as previously reported UiO-66 MOFs (Molavi et al., 2018). Consequently, it can conclude that the anchoring MOFs onto the PVDF membrane and the introduction of quaternary ammonium groups would not change the crystal structure of UiO-66 framework significantly.

XPS analysis of UiO-66- $\mathrm{NMe}_{3}{ }^{+} \mathrm{MMM}$ was carried out to define the surface chemical composition. The survey of the prepared UiO-66$\mathrm{NMe}_{3}{ }^{+}$MMM was illustrated in Fig. S3a. The peaks of C 1s, Zr 3d, O 1s, $\mathrm{N} 1 \mathrm{~s}, \mathrm{Cl} 2 \mathrm{p}$ and $\mathrm{F} 1 \mathrm{~s}$ appeared at 286.1, 182.8, 531.6, 399.6, 196.8 and $688.1 \mathrm{eV}$, respectively. The high resolution XPS spectrum for Zr 3d (Fig. $\mathrm{S} 3 \mathrm{~b}$ ) indicated the double binding energy position of the $\mathrm{Zr} 3 \mathrm{~d} \mathrm{3/2}$ and $\mathrm{Zr} 3 \mathrm{~d} 5 / 2$ peaks were around $185.0 \mathrm{eV}$ and $182.7 \mathrm{eV}$, respectively. The data are consistent with the reported values for UiO-66 (Yang et al., 2019). The binding peaks of $C$ 1s (Fig. S3c) at 290.0, 288.5, 285.4 and $284.5 \mathrm{eV}$ were corresponding to carbon atoms in different functional groups, namely $\mathrm{CH}_{2}-\mathrm{CF}_{2}, \mathrm{C}-\mathrm{O}, \mathrm{C}-\mathrm{C}$ and $\mathrm{C}-\mathrm{NMe}_{3}$, respectively (Liu et al., 2015; Akashi and Kuroda, 2014). Additionally, the N 1s XPS spectrum (Fig. S3d) showing the binding energies at 399.0 and 400.4 $\mathrm{eV}$, could be attributed to the nitrogen atoms in $\mathrm{Ph}-\mathrm{NMe}_{3}$ and $\mathrm{C}-\mathrm{N}$ groups (Liu et al., 2015; Xu et al., 2013).

To further confirm the functionalization of quaternary amine groups, UiO-66- $\mathrm{NH}_{2} \mathrm{MMM}$ and UiO-66- $\mathrm{NMe}_{3}{ }^{+} \mathrm{MMM}$ were digested in $\mathrm{d}_{6}$-DMSO with HF solution to obtain NMR data. The undissolved PVDF membrane were decanted to attain a clarify mixture. As depicted in the $1 \mathrm{H}$ NMR spectrum of UiO-66- $\mathrm{NH}_{2}$ (Fig. S4a), proton peaks at 7.04, 7.38 and $7.78 \mathrm{ppm}$ are related to 2-amino terephthalate ligand (Molavi et al., 2018; Goh et al., 2015). The common product of methylation of primary amine is a mixture of secondary, tertiary and quaternary amine. Therefore, newly appeared proton peaks $(\delta=7.09$ for secondary amine, $\delta=7.10$ for tertiary amine and $\delta=7.22$ for quaternary amine (Lebleu et al., 2014) in UiO-66- $\mathrm{NMe}_{3}{ }^{+}$spectra (Fig. S4b) suggested the successful functionalization of UiO-66- $\mathrm{NH}_{2}$. In addition, functionalization degree of quaternary amine groups was calculated about $53 \%$ from $1 \mathrm{H}$ NMR spectroscopy, which is calculated from the peak area proportion of the quaternary amine in total newly appearing proton peaks for secondary amine, tertiary amine and quaternary amine, according to that reported (Lebleu et al., 2014).

The surface charge $(\delta)$ of the UiO-66- $\mathrm{NMe}_{3}{ }^{+} \mathrm{MMM}$ was measured by an acid-base titration method. A piece of the membrane $(0.073 \mathrm{~g})$ was soaked in $100 \mathrm{~mL} \mathrm{CO}_{2}$ free aqueous solution. $0.01 \mathrm{M} \mathrm{HCl}$ or $\mathrm{NaOH}$ was dripped into the above mixture, and the volume of added acid or base and the solution $\mathrm{pH}$ values were recorded. The surface charge was calculated via the following equation,

$\delta=\frac{\left(C_{A}-C_{B}+\left[\mathrm{OH}^{-}\right]+\left[H^{+}\right]\right) \mathrm{F}}{m}$

where $C_{\mathrm{A}}$ and $C_{\mathrm{B}}$ represent the added concentration of $\mathrm{HCl}$ and $\mathrm{NaOH}$ (mol/L) to a certain $\mathrm{pH}$ value, respectively; $\left[\mathrm{OH}^{-}\right]$and $\left[\mathrm{H}^{+}\right]$are the concentrations of $\mathrm{OH}^{-}$and $\mathrm{H}^{+}$(mol/L), respectively; $\mathrm{F}$ is the faraday's constant $(96,490 \mathrm{C} / \mathrm{mol}) ; m$ is the dosage of membrane $(\mathrm{g} / \mathrm{L})$. The calculated point of zero charge from the equation is 9.4 , indicating the UiO-66- $\mathrm{NMe}_{3}{ }^{+} \mathrm{MMM}$ induced positive charge when the solution $\mathrm{pH}$ was lower than 9.4.

The pore size of UiO-66- $\mathrm{NMe}_{3}{ }^{+}$MMM was estimated by bubble point measurement, and the mean pore size was attained as about $3.04 \mu \mathrm{m}$. These micron pores might be fabricated by the integrated MOFs via polymer binders, and thereby result from the membrane structure. Then, BET analysis was also carried out. As listed in Table S2, the specific surface areas, cumulative pore volume and average pore diameter of UiO-66- $\mathrm{NH}_{2} \mathrm{MMM}$ were $339.7 \mathrm{~m}^{2} / \mathrm{g}, 0.49 \mathrm{~mL} / \mathrm{g}$ and $5.88 \mathrm{~nm}$, respectively, while those of UiO-66- $\mathrm{NMe}_{3}{ }^{+} \mathrm{MMM}$ were $318.3 \mathrm{~m}^{2} / \mathrm{g}, 0.43 \mathrm{~mL} / \mathrm{g}$ and $6.12 \mathrm{~nm}$, respectively. The average pore diameter of $5.88 \mathrm{~nm}$ and $6.12 \mathrm{~nm}$ was calculated by $4 \mathrm{~V} / \mathrm{A}$ equation, where the values refer to $\mathrm{V}$ (volume) and $\mathrm{A}$ (area). This equation assumes that the pores must be cylindrical and homogeneous in diameter, which is an ideal limiting condition. As seen in Fig. S5, the adsorption/ desorption isotherms of UiO-66- $\mathrm{NH}_{2}$ MMM (a) and UiO-66- $\mathrm{NMe}_{3}{ }^{+}$ MMM (b) could be classified into type IV, indicating the existence of mecroporous, which could be attributed to the interparticle separation (Das et al., 2011; Bhunia et al., 2014). The presence of hysteresis loop might be due to the capillary condensation within the materials. The pore size distribution plots based on the density functional theory (DFT) were adopted to measure the pore size, and the sharpest peak appeared in the pore size distribution plots could indicate the average pore

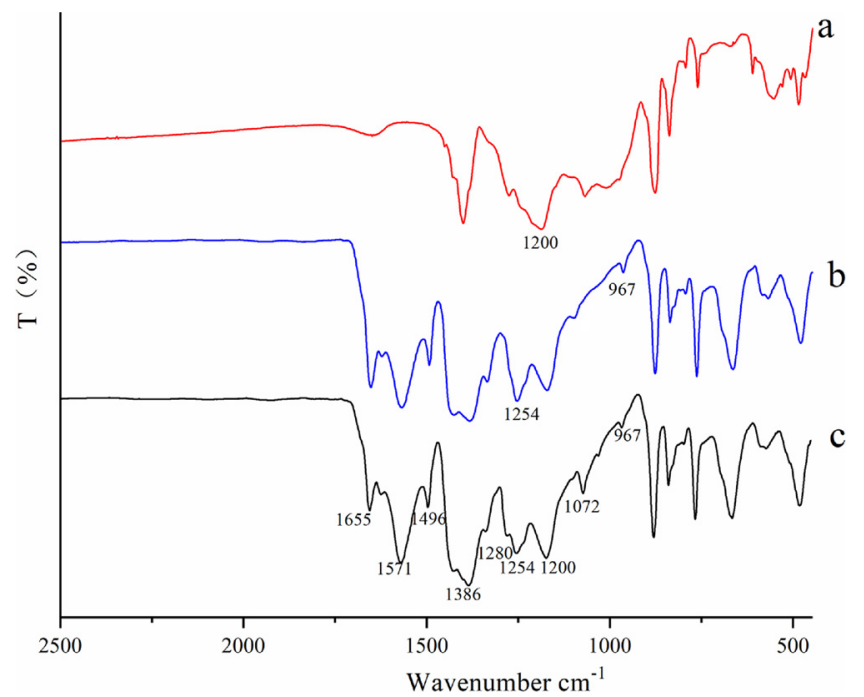

Fig. 3. FTIR spectra of PVDF (a), UiO-66- $\mathrm{NH}_{2} \mathrm{MMM}$ (b) and UiO-66-NMe ${ }_{3}{ }^{+}$ MMM (c). 


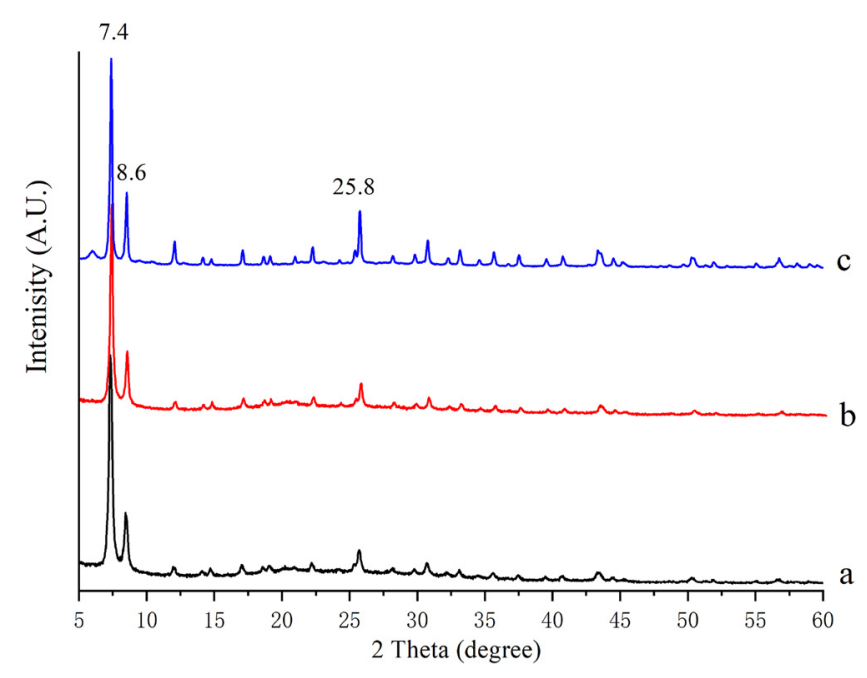

Fig. 4. XRD patterns of UiO-66- $\mathrm{NH}_{2} \mathrm{MMM}$ (a) and UiO-66-NMe ${ }_{3}{ }^{+} \mathrm{MMM}$ (b), and simulated XRD pattern of UiO-66 crystal (c).

diameter of this material (He et al., 2017). As shown in Fig. S5, the pore diameter of UiO-66- $\mathrm{NH}_{2}$ MMM (a1) and UiO-66- $\mathrm{NMe}_{3}{ }^{+} \mathrm{MMM}$ (b1) were about 0.56 and $0.62 \mathrm{~nm}$, respectively, which is consistent with the reported value for UiO-66 MOFs (Katz et al., 2013). The nanoscale pores were probably present within MOFs crystals, that is, they should belong to the MOFs structure. Very possibly, the formation of quaternary ammonium groups enlarged the pore size and led to the slight reduction of specific surface area of UiO-66- $\mathrm{NMe}_{3}{ }^{+} \mathrm{MMM}$ comparing with UiO-66- $\mathrm{NH}_{2}$ MMM. So, the two MMM materials were highly porous. The high porosity of UiO-66- $\mathrm{NMe}_{3}{ }^{+}$MMM facilitated mass transfer of target analytes from sample solutions into the adsorbent (Li et al., 2018).

The mechanical stability of UiO-66- $\mathrm{NMe}_{3}{ }^{+} \mathrm{MMM}$ was assessed by tensile test. Comparing with the ultimate tensile strength (UTS) value of reported pure PVDF membrane (1.17 MPa) (Sierke and Ellis, 2019), the UTS of UiO-66- $\mathrm{NMe}_{3}{ }^{+} \mathrm{MMM}$ had a slight lower value as approximately $1.04 \mathrm{MPa}$. Tensile data proved the prepared UiO-66- $\mathrm{NMe}_{3}{ }^{+} \mathrm{MMM}$ was robust enough for DME process and resistant to physical shock.

The hydrophilicity of UiO-66- $\mathrm{NMe}_{3}{ }^{+} \mathrm{MMM}$ and pristine PVDF membrane was evaluated by surface water contact angle (CA) measurement. As observed in Fig. S6, the CA degrees for pristine PVDF membrane and UiO-66- $\mathrm{NMe}_{3}{ }^{+} \mathrm{MMM}$ were $112^{\circ}$ and $90^{\circ}$, respectively. This decrement verified that the surface hydrophilicity of the membrane was improved by the addition of MOFs. Possibly, the introduction of hydrophilic $\mathrm{NMe}_{3}{ }^{+}$groups into MOFs would enhance the hydrophilicity of the prepared MMM.

\subsection{Optimization of UiO-66- $\mathrm{NMe}_{3}{ }^{+} \mathrm{MMM}$ based DME conditions}

To obtain the highest extraction efficiency and verify the adsorption mechanism, the influence of some important factors including dosage ratio of MOFs in MOF-MMM, extraction conditions (e.g., pH, time, coexist anions and ionic strength) and desorption conditions (e.g., type and volume of eluent, and time) were investigated as follows.

\subsubsection{Effect of the dosage ratio of $M O F / P V D F$}

The integration of MOF particles with an appropriate amount into the MMM plays a significant impact on the reactive binding sites of the final material. Thus, the efficacy of the dosage ratio of MOF/PVDF was investigated. Different amounts of MOFs such as 20, 30, 40, 50, 60 and $80 \mathrm{mg}$ were mixed with $40 \mathrm{mg}$ of PVDF to obtain the MOF/PVDF dosage ratio of $1: 2$ to 2:1. As displayed in Fig. 5A, by the increasing amount of MOF from 20 to $60 \mathrm{mg}$, the extraction performances of six PCAs were elevated and then slightly decreased at $80 \mathrm{mg}$. This phenomenon might be due to that the excess loading of MOFs on PVDF based matrix led to the irregular functionalization of UiO-66- $\mathrm{NH}_{2} \mathrm{MMM}$, which hindered the extraction of PCAs in water samples. Therefore, $60 \mathrm{mg}$ of UiO-66$\mathrm{NH}_{2}$ that is equivalent to MOF/PVDF dosage ratio of 1.5:1 was set for further experiments.

\subsubsection{Effect of solution $p H$}

The $\mathrm{p} K_{\mathrm{a}}$ values of six PCAs (Fig. S1) represent these compounds can easily be ionized in aqueous solutions, thus, the solution $\mathrm{pH}$ would directly affect the ionization degree of PCAs. Meanwhile, solution $\mathrm{pH}$ also determines the surface charge of the UiO-66- $\mathrm{NMe}_{3}{ }^{+} \mathrm{MMM}$. Hence, the solution $\mathrm{pH}$ was set with $0.01 \mathrm{M}$ of $\mathrm{HCl}$ or $\mathrm{NaOH}$ in the range of 3.0-8.0. From Fig. 5B, the extraction efficiencies of six PCAs remained almost constant at the same level within $\mathrm{pH}$ 3.0-6.0, and then diminished at $\mathrm{pH}>6.0$. At $\mathrm{pH}$ of 3.0, PCAs were in free acid form and the main adsorption mechanism might be $\pi-\pi$ conjugation of the benzene rings between UiO-66 frameworks and PCAs, along with cation- $\pi$ bonding among the natural form of PCAs and cationic quaternary amine group in UiO-66- $\mathrm{NMe}_{3}{ }^{+}$. It should be pointed out that cation- $\pi$ boning can be formed between the cationic group and $\pi$-electron-rich aromatic structure (Zhao et al., 2017). By increasing the pH values, PCAs molecules became natural and the electron density in benzene ring was enhanced, so $\pi-\pi$ interactions were reduced. The zeta potential of UiO66- $\mathrm{NMe}_{3}{ }^{+} \mathrm{MMM}$ was calculated as about 9.4. Therefore, the membrane had positive charge within the $\mathrm{pH}$ range of 3.0-6.0, electrostatic interactions were boosted when PCAs became deprotonated, and thus the adsorption performance was approximately unchanged at this $\mathrm{pH}$ range. With the continuous increase of $\mathrm{pH}$ value, more anionic $\mathrm{OH}^{-}$ species existed in the solution and would compete for interaction with the positive charges in the binding sites of UiO-66- $\mathrm{NMe}_{3}{ }^{+} \mathrm{MMM}$, leading to decrease of extraction efficiencies. Consequently, the further experiments were conducted without adjusting $\mathrm{pH}$.

\subsubsection{Effect of extraction time}

Sufficient extraction time is essential for adsorbent to capture target analytes until extraction equilibrium. Herein, different extraction times within the range of 10-40 min were investigated. As shown in Fig. 5C, the maximum extraction efficiencies of six PCAs were attained when the extraction time was $30 \mathrm{~min}$, and reached a plateau. Thus, $30 \mathrm{~min}$ was selected as the optimum extraction time.

\subsubsection{Effect of desorption conditions}

Desorption conditions including type and volume of eluent solvent and desorption time are significant parameters that can substantially influence the extraction recovery. Methanol has high elution strength and can freely dissolve PCAs. On the other hand, addition of an appropriate concentration of ammonia to the organic solvent was proved to be beneficial for facilitation of eluting such acidic analytes (Zhang et al., 2018). In this regards, four concentrations of ammonia in methanol at $0.5 \%, 1 \%, 1.5 \%$ and $2 \%,(\mathrm{v} / \mathrm{v})$ were used to investigate the effect of desorption solvent on the extraction efficiencies. The results showed that the extraction efficiencies of six PCAs were improved with the increasing ammonia concentration from $0.5 \%$ to $1 \%$, followed by decreasing at higher ammonia concentrations (Fig. 5D). The decline of extraction efficiencies at high ammonia concentrations might be owing to that the excessive ammonia destroyed the function groups in UiO-66$\mathrm{NMe}_{3}{ }^{+}$MMM. Thus, $1 \%$ ammonia/methanol was used for further studies.

Since the fresh solvent can desorb much more analyte, the eluent process was conducted twice. $1 \times 2,1.5 \times 2,2 \times 2$, and $2.5 \times 2 \mathrm{~mL}$ of $1 \%$ ammonia/methanol were used to investigate the optimum eluent volume. According to the obtained results (Fig. 5E), the maximum extraction efficiencies were achieved at $5 \mathrm{~mL}$ of desorption solvent. Therefore, $2.5 \times 2 \mathrm{~mL}$ of $1 \%$ ammonia/methanol was adopted for the remaining experiments.

The influence of desorption time in the range of 5-30 min was 

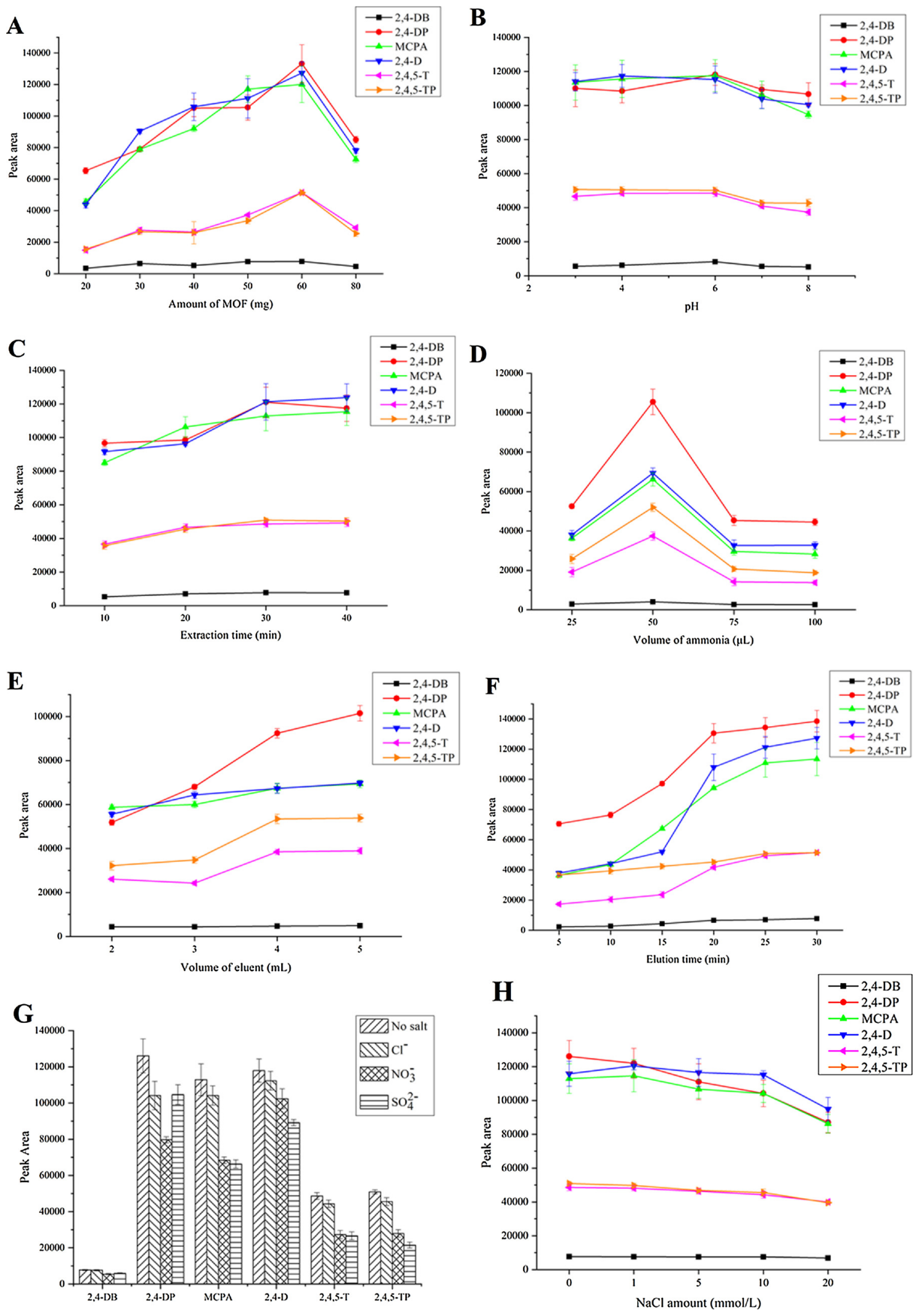

Fig. 5. Effects of (A) dosage ratio of MOF/PVDF, (B) sample $\mathrm{pH}$, (C) extraction time, (D) ammonia concentration in eluent, (E) volume of eluent, (F) desorption time, (G) coexistent anions, and (H) ionic strength on the extraction efficiency of UiO-66- $\mathrm{NMe}_{3}{ }^{+}$DME for PCAs. 
Table 1

Related analytical parameters of UiO-66- $\mathrm{NMe}_{3}{ }^{+} \mathrm{MMM}$ based DME coupled with UHPLC-MS/MS determination for PCAs.

\begin{tabular}{llllll}
\hline PCAs & Regression equation $^{\mathrm{a}}$ & $\begin{array}{l}\text { Correlation } \\
\text { coefficient }(r)\end{array}$ & $\begin{array}{l}\text { Linear } \\
\text { range } \\
(\mathrm{ng} / \mathrm{L})\end{array}$ & $\begin{array}{l}\text { LOD } \\
(\mathrm{ng} / \mathrm{L})\end{array}$ & $\begin{array}{l}\text { LOQ } \\
(\mathrm{ng} / \mathrm{L})\end{array}$ \\
\hline 2,4-DB & $y=40.872 x-55.256$ & 0.9941 & $5-400$ & 0.59 & 1.95 \\
$2,4-\mathrm{DP}$ & $y=836.72 x-302.31$ & 0.9986 & $0.4-400$ & 0.036 & 0.11 \\
MCPA & $y=805.62 x-739.63$ & 0.9995 & $0.4-400$ & 0.036 & 0.11 \\
2,4-D & $y=830.74 x+156.51$ & 0.9976 & $0.4-400$ & 0.031 & 0.09 \\
$2,4,5-\mathrm{T}$ & $y=284.23 x-142.31$ & 0.9946 & $0.4-400$ & 0.12 & 0.38 \\
$2,4,5-\mathrm{TP}$ & $y=265.06 x-254.31$ & 0.9997 & $0.4-400$ & 0.12 & 0.36 \\
\hline
\end{tabular}

${ }^{\mathrm{a}} x=$ concentration (ng/L), $y=$ peak area.

tested. As seen in Fig. 5F, the extraction yields of six PCAs were enhanced and then reached a plateau at about $30 \mathrm{~min}$. Therefore, desorption time was set at $30 \mathrm{~min}$ to fully elute the adsorbed analytes.

\subsubsection{Effect of coexistent anions}

Coexistent anions in the water samples might competitively occupy the binding sites of the UiO-66- $\mathrm{NMe}_{3}{ }^{+}$MMM. Therefore, the effect of three types of anions with different size and charge (chloride, nitrate, and sulfate at $10 \mathrm{mmol} / \mathrm{L}$ ) on the extraction of PCAs was investigated and the experimental results were presented in Fig. 5G. As seen, the divalent ion (sulfate) had a larger negative effect than monovalent ions (chloride, nitrate). As above-discussed in 3.3.2. Effect of solution $\mathrm{pH}$, electrostatic interactions dominated the extraction process. Thus, divalent sulfate exhibited higher competitive than monovalent ions. On the other hand, the p-electron clouds of planar nitrates can interact with the benzene ring of UiO-66-NMe ${ }^{3+}$ (Slingsby and Pohl, 1988; Kang et al., 2018). Accordingly, the nitrate anions occupied more adsorption sites of UiO-66- $\mathrm{NMe}^{3+}$ compared with chloride anions, which led to larger negative effect on the adsorption efficiency. Overall, the presence of anions resulted in the decline of extraction efficiencies. Hence, further experiments were performed without adding anions.

\subsubsection{Effect of ionic strength}

The ionic strength of sample solution can influence the affinity/ sensitivity of absorbents and thereby adsorption performance toward target analytes by salting-out and competitive effects (Otalvaro et al., 2019; Li et al., 2019). Accordingly, the effect of ionic strength on the extraction efficiency was investigated by adding different concentrations of $\mathrm{NaCl}(0,1,5,10,20 \mathrm{mmol} / \mathrm{L})$, which is commonly used for ionic strength examination (Otalvaro et al., 2019). As observed from Fig. $5 \mathrm{H}$, the extraction efficiencies of almost all the tested PCAs were decreased with the concentration increase of $\mathrm{NaCl}$, except 2,4-D and MCPA showed slight increase of efficiencies merely within $0-1 \mathrm{mmol} / \mathrm{L} \mathrm{NaCl}$, as well as 2,4-DB showed nearly unchanged efficiencies. This phenomenon can be explained as that the dissociated $\mathrm{NaCl}$ generated free anions, which competitively occupied some adsorption sites of UiO-66- $\mathrm{NMe}_{3}{ }^{+} \mathrm{MMM}$, impeding the adsorption of PCAs by the MMM. This also could confirm that the adsorption of anionic PCAs onto the cationic MMM was mainly driven by electrostatic interactions. Herein, in general, ionic strength hadn't positive influence on the adsorption performance, so, subsequent experiments were conducted without addition of $\mathrm{NaCl}$.

\subsection{Possible adsorption mechanism of the UiO-66- $\mathrm{NMe}_{3}{ }^{+} \mathrm{MMM}$ towards PCAs}

As above experimental results suggested, electrostatic interactions were the main adsorption mechanism between anionic PCAs and cationic UiO-66- $\mathrm{NMe}_{3}{ }^{+}$MMM. The $\pi-\pi$ conjugation and cation- $\pi$ bonding between UiO- 66 frameworks and PCAs also benefited the adsorption behavior. The possible adsorption mechanism was schematically shown in Fig. S7. In order to confirm this fact and demonstrate the effectiveness of functionalized quaternary ammonium groups, the adsorption performance of unmodified UiO-66- $\mathrm{NH}_{2}$ MMM was compared with that of the UiO-66- $\mathrm{NMe}_{3}{ }^{+} \mathrm{MMM}$ under the same above-optimized DME conditions, by using DME efficiencies as an index. As seen in Fig. S8, the extraction efficiencies of DME for all the PCAs were significantly improved by UiO-66- $\mathrm{NMe}_{3}{ }^{+} \mathrm{MMM}$. It is well known that specific surface area of an adsorbent has a significant influence on the adsorption performance. Generally, the adsorbent with larger specific surface area has higher adsorption capacity; however, this phenomenon only occurs when Vander Waals interaction is the main adsorption mechanism (Sarker et al., 2018). In our present study, PCAs were adsorbed on the UiO-66- $\mathrm{NMe}_{3}{ }^{+} \mathrm{MMM}$ via dominate electrostatic interactions along with $\pi-\pi$ conjugations and cation- $\pi$ bonding, and therefore, the adsorption capacity wasn't affected by the lower surface area but attained higher values. Consequently, the adsorption mechanism of UiO-66$\mathrm{NMe}_{3}{ }^{+}$MMM could be attributed to the important role of $\mathrm{NMe}_{3}{ }^{+}$, indicating the iMOFs were in favor of adsorption.

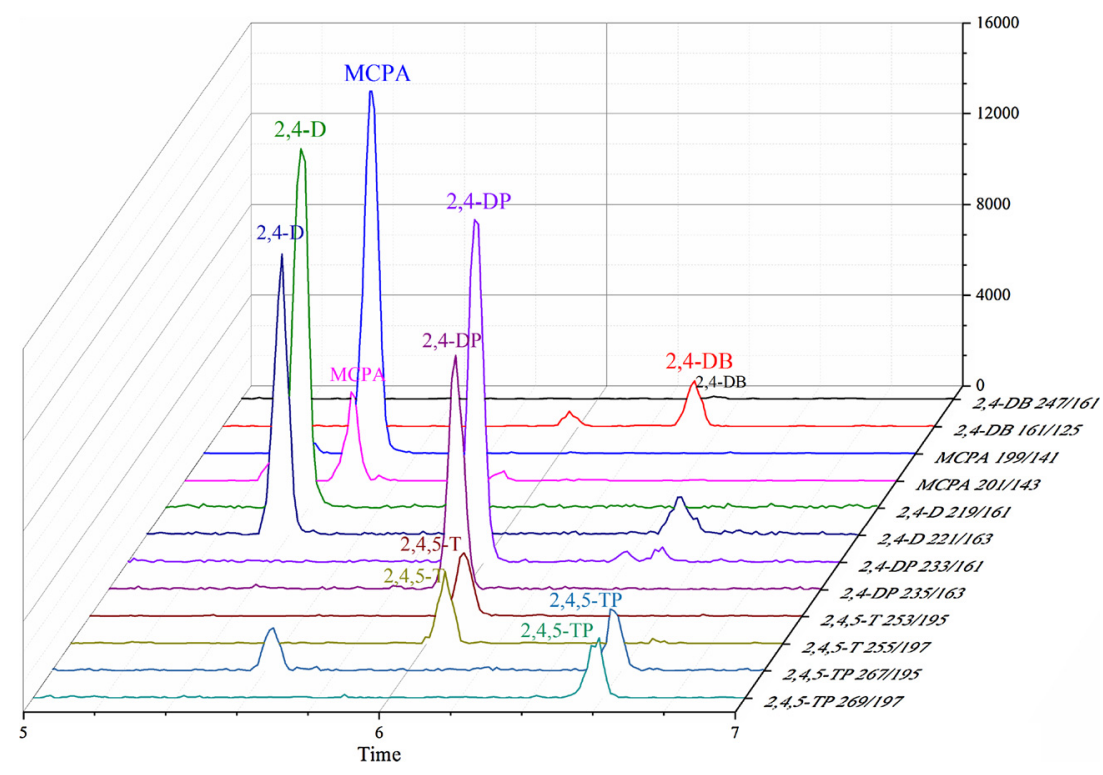

Fig. 6. Chromatograms of six PCAs spiked at $50 \mathrm{ng} / \mathrm{L}$ in sewage water sample. 


\subsection{Analytical performance of the DME-UHPLC-MS/MS}

Under the optimized extraction conditions, the established method was validated by evaluating several quantitative characteristics. As illustrated in Table 1 , the method presented good linearity within the concentration ranges of $5-400 \mathrm{ng} / \mathrm{L}$ for $2,4-\mathrm{DB}$ at six spiking concentration levels (i.e., 5, 10, 50, 100, 200 and $400 \mathrm{ng} / \mathrm{L}$ ) and 0.4-400 ng/L for 2,4-DP, MCPA, 2,4-D, 2,4,5-T and 2,4,5-TP at seven spiking concentration levels (i.e., 0.4, 5, 10, 50, 100, 200 and $400 \mathrm{ng} /$ L). Calibration curves were obtained by plotting the peak areas $(y)$ of PCAs versus their concentrations $(x)$. The obtained correlation coefficients $\left(r^{2}\right)$ for six PACs ranged from 0.9941 to 0.9997 . The limits of detection (LODs) and the limits of quantitation (LOQs) were calculated based on analyte signal to background noise ratio of 3 and 10 in the spiked water sample, respectively. As seen in Table 1, the LODs and LOQs of six PACs ranged from 0.03 to $0.59 \mathrm{ng} / \mathrm{L}$ and $0.11-1.59 \mathrm{ng} / \mathrm{L}$, respectively. The precision of the proposed method was assessed by relative standard deviations (RSDs) of peak areas at three concentration levels $(8,50$ and $100 \mathrm{ng} / \mathrm{L})$. As listed in Table S3, the intra-day $(n=6)$ and inter-day $(n=6)$ RSDs were in the range of $2.30-9.30 \%$ and 3.26-9.79\%, respectively. It can be concluded that the developed analytical method possesses supreme sensitivity and precision for simultaneous determination of PCAs at trace levels.

\subsection{Method application for determination of PCAs in real water samples}

The applicability of the developed UiO-66- $\mathrm{NMe}_{3}{ }^{+}$MMM-DME coupled with UPLC-MS/MS method was further evaluated. Two water samples from sewage and reservoir were analyzed. The chromatograms of spiked sewage samples at $50.0 \mathrm{ng} / \mathrm{L}$ were illustrated in Fig. 6 and the results were listed in Table 2. Trace amounts of 2,4-DB, 2,4-DP, MCPA and 2,4-D were detected in sewage water samples, and no PCAs were detected in reservoir water samples. Two levels of spiked samples at $8.0 \mathrm{ng} / \mathrm{L}$ and $50.0 \mathrm{ng} / \mathrm{L}$ were used to investigate the extraction recoveries of the developed method. Satisfactory recoveries for all the six PCAs were obtained in the range of 80.06-112.42\% for sewage water sample and $80.64-117.40 \%$ for reservoir water sample. The above results demonstrated the developed method had high applicability for influential enrichment and detection of trace PCAs in complex water samples.

\subsection{Method performance comparison}

Analytical performance of the developed UiO-66- $\mathrm{NMe}_{3}{ }^{+} \mathrm{MMM}$ based DME method was compared with some reported methods for the analysis of the PCAs. For fair judgment, we have tried to choose the methods in which the sensitivity of their detectors is almost similar. As seen in Table 3, the current method offers much lower LODs than those of existing methods. Moreover, in some pretreatment methods (Ma et al., 2019b; Gao et al., 2014; Wang et al., 2017), pH adjustment is needed which increased the experimental steps and time consuming. The water samples can be analyzed without adjusting $\mathrm{pH}$ in our developed method, thus, the pretreatment operation is simplified. Comparing with the most used real water samples, such as river water and tap water, our method can be applied to more complex sewage water samples, indicating higher specificity and affinity of the UiO-66- $\mathrm{NMe}_{3}{ }^{+}$ MMM towards PCAs.

\section{Conclusions}

In the present work, a novel cationic UiO-66- $\mathrm{NMe}_{3}{ }^{+} \mathrm{MMM}$ was successfully synthesized by a facile method. The prepared UiO-66$\mathrm{NMe}_{3}{ }^{+}$MMM was fully characterized and subsequently applied for enrichment of trace amounts of polar PCAs from water samples. Combined with UHPLC-MS/MS, under the optimized extraction conditions, the developed method supplied excellent sensitivity with LODs at $\mathrm{pg} / \mathrm{L}$ levels. The feasibility of the developed analytical method was verified by quantification analysis of PCAs in complex water samples, such as sewage and reservoir water. Satisfactory precision and accuracy were achieved by this established pretreatment method, indicating that the proposed UiO-66- $\mathrm{NMe}_{3}{ }^{+} \mathrm{MMM}$ is a highly efficient, selective and reliable material for the enrichment of polar PCAs at ultra-trace concentrations.

\section{CRediT authorship contribution statement}

Gege Wu: Methodology, Data curation, Formal analysis, Writing original draft. Jiping Ma: Supervision, Conceptualization, Methodology, Funding acquisition. Shasha Wang: Formal analysis, Validation. Huining Chai: Investigation. Liang Guo: Investigation. Jinhua Li: Writing - review \& editing. Abbas Ostovan: Writing - original draft. Yafeng Guan: Project administration. Lingxin Chen: Conceptualization, Writing - review \& editing.

\section{Declaration of Competing Interest}

The authors declare that they have no known competing financial interests or personal relationships that could have appeared to influence the work reported in this paper.

\section{Acknowledgments}

This work was financially supported by the National Natural Science Foundation of China (21976099, 21876199), the Natural Science Foundation of Shandong Province of China (ZR2019MB046), the Chinese Academy of Sciences President's International Fellowship Initiative (2020PC0083), and the Taishan Scholar Project Special Funding (ts20190962).

Table 2

Determination of PCAs and method recoveries in real water samples.

\begin{tabular}{|c|c|c|c|c|c|}
\hline \multirow[t]{2}{*}{ PCAs } & \multirow{2}{*}{$\begin{array}{l}\text { Spiked ( } \mu \mathrm{g} / \\
\mathrm{L})\end{array}$} & \multicolumn{2}{|c|}{ Sewage water ${ }^{\mathrm{a}}$} & \multicolumn{2}{|c|}{ NC reservoir water ${ }^{b}$} \\
\hline & & $\begin{array}{l}\text { Found (ng/ } \\
\text { L) }\end{array}$ & Recovery (\%) & $\begin{array}{l}\text { Found (ng/ } \\
\text { L) }\end{array}$ & Recovery (\%) \\
\hline \multirow[t]{3}{*}{$2,4-\mathrm{DB}$} & 0 & 14.87 & & $\mathrm{ND}^{\mathrm{c}}$ & \\
\hline & 8 & 21.28 & 80.19 & 9.39 & 117.40 \\
\hline & 50 & 61.68 & 93.62 & 51.17 & 102.33 \\
\hline \multirow[t]{3}{*}{ 2,4-DP } & 0 & 5.03 & & ND & \\
\hline & 8 & 12.15 & 88.95 & 6.81 & 85.10 \\
\hline & 50 & 58.27 & 106.48 & 43.47 & 86.94 \\
\hline \multirow[t]{3}{*}{ MCPA } & 0 & 5.54 & & ND & \\
\hline & 8 & 13.15 & 95.11 & 8.51 & 106.39 \\
\hline & 50 & 59.30 & 107.52 & 43.40 & 86.80 \\
\hline \multirow[t]{3}{*}{ 2,4-D } & 0 & 11.15 & & ND & \\
\hline & 8 & 17.83 & 83.43 & 7.36 & 92.03 \\
\hline & 50 & 67.36 & 112.42 & 47.41 & 94.82 \\
\hline \multirow[t]{3}{*}{$2,4,5-\mathrm{T}$} & 0 & ND & & ND & \\
\hline & 8 & 7.22 & 90.29 & 6.81 & 85.15 \\
\hline & 50 & 42.13 & 84.25 & 41.74 & 83.48 \\
\hline \multirow[t]{3}{*}{ 2,4,5-TP } & 0 & ND & & $\mathrm{ND}$ & \\
\hline & 8 & 7.93 & 99.08 & 6.53 & 81.67 \\
\hline & 50 & 40.03 & 80.06 & 40.32 & 80.64 \\
\hline
\end{tabular}

${ }^{\text {a }}$ From Licunsewage treatment plant.

b From NuoCheng Reservoir.

c Not detected. 
Appendix A. Supplementary data

Supplementary material related to this article can be found, in the online version, at doi:https://doi.org/10.1016/j.jhazmat.2020.122556.

\section{References}

Akashi, N., Kuroda, S., 2014. Protein immobilization onto poly(vinylidene fluoride) microporous membranes activated by the atmospheric pressure low temperature plasma. Polymer 55, 2780-2791.

Arcaute, C.R., Soloneski, S., Larramendy, M.L., 2016. Toxic and genotoxic effects of the 2,4-dichlorophenoxyacetic acid (2,4-D)-based herbicide on the Neotropical fish Cnesterodon decemmaculatus. Ecotoxicol. Environ. Saf. 128, 222-229.

Bhunia, S., Chatterjee, N., Das, S., Saha, K.D., Bhaumik, A., 2014. Novel porous polyurea network showing aggregation induced white light emission, applications as biosensor and scaffold for drug delivery. ACS Appl. Mater. Interfaces 6, 22569-22576.

Chen, K., Wu, C.D., 2019. Designed fabrication of biomimetic metal-organic frameworks forcatalytic applications. Coord. Chem. Rev. 378, 445-465.

Das, S.K., Bhunia, M.K., Seikh, M.M., Dutta, S., Bhaumik, A., 2011. Highly porous Co(II)salicylate metal-organic framework: synthesis, characterization and magnetic properties. Dalton Trans. 40, 2932-2939.

Denny, M.S., Cohen, S.M., 2015. In situ modification of metal-organic frameworks in mixed-matrix membranes. Angew. Chem. Int. Ed. 54, 9029-9032.

Farha, O.K., Spokoyny, A.M., Mulfort, K.L., Hawthorne, M.F., Mirkin, C.A., Hupp, J.T., 2007. Synthesis and hydrogen sorption properties of carborane based metal - organic framework materials. J. Am. Chem. Soc. 129, 12680-12681.

Fu, F.F., Xiao, L.X., Wang, W., Xu, X.Q., Xu, L.J., Qi, G.M., Chen, G.N., 2009. Study on the degradation of 2,4-dichlorophenoxyacetic acid (2,4-D) and 2-methyl-4-chloro-phenoxyacetic sodium (MCPA sodium) in natural agriculture-soils of Fuzhou, China using capillary electrophoresis. Sci. Total Environ. 407, 1998-2003.

Gao, N., Cai, K., Guo, X.C., Zhang, Y.P., Yang, S., Hu, D.Y., 2014. Analysis of MCPA and TCP in water by liquid chromatography-ion trap-electrospray tandem mass spectrometry. J. Environ. Anal. Chem. 94, 594-605.

Gao, G.H., Li, S., Li, S.J., Zhao, L., Wang, T., Hou, X.H., 2018. Development and application of vortex-assisted membrane extraction based on metal-organic framework mixed-matrix membrane for the analysis of estrogens in human urine. Anal. Chim. Acta 1023, 35-43.

Ghambarian, M., Behbahani, M., Esrafili, A., Sobhi, H.R., 2017. Application of a dispersive solid-phase extraction method using an amino-based silica-coated nanomagnetic sorbent for the trace quantification of chlorophenoxyacetic acids in water samples. J. Sep. Sci. 40, 3479-3486.

Goh, T.W., Xiao, C.X., Maligal-Ganesh, R.V., Li, X.L., Huang, W.Y., 2015. Utilizing mixedlinker zirconium based metal-organic frameworks to enhance the visible light photocatalytic oxidation of alcohol. Chem. Eng. Sci. 124, 45-51.

He, Y.R., Tang, Y.P., Ma, D.C., Chung, T.S., 2017. UiO-66 incorporated thin-film nanocomposite membranes for efficient selenium and arsenic removal. J. Membr. Sci. 541, 262-270.

Howarth, A.J., Liu, Y., Hupp, J.T., Farha, O.K., 2015. Metal-organic frameworks for applications in remediation of oxyanion/cation-contaminated water. CrystEngComm $17,7245-7253$.

Jasmina, H.C., Søren, J., Unni, O., Nathalie, G., Carlo, L., Silvia, B., Karl, P.L., 2008. A new zirconium organic building brick forming metal organic frameworks with exceptional stability. J. Am. Chem. Soc. 130, 13850-13851.

Jia, Z.Y., Cheng, J., Song, C.H., Hu, N., Zhou, W., Suo, Y.R., Sun, Z.W., You, J.M., 2019. A highly sensitive and selective method for determination of phenoxy carboxylic acids from environmental water samples by dispersive solid phase extraction coupled with ultrahigh performance liquid chromatography-tandem mass spectrometry. Talanta 191, 313-323.

Kang, Y., Chen, W.C., Zhang, H., Sun, L., Wu, T., Du, Y.P., 2018. Real-time preparation of surface enhanced Raman scattering substrate for on-line analysis of aromatic molecules in capillary. Microchem. J. 137, 15-21.

Katz, M.J., Brown, Z.J., Colon, Y.J., Siu, P.W., Scheidt, K.A., Snurr, R.Q., Hupp, J.T., Farha, O.K., 2013. A facile synthesis of UiO-66, UiO-67 and their derivatives. Chem. Commun. 49, 9449-9451.

Lebleu, T., Ma, X.L., Maddaluno, J., Legros, J., 2014. Selective monomethylation of primary amines with simple electrophiles. Chem. Commun. 50, 1836-1838.

Li, H., Sun, Y., Yuan, Z.Y., Zhu, Y.P., Ma, T.Y., 2018. Titanium phosphonate based metal-organic frameworks with hierarchical porosity for enhanced photocatalytic hydrogen evolution. Angew. Chem. Int. Ed. 57, 3222-3227.

Li, R.P., Tang, Y.M., Li, X., Tang, C.J., Zhu, Y.X., Wang, S.L., Lin, C.Y., 2019. Adsorption behavior of tungstate on montmorillonite as a function of $\mathrm{pH}$, ionic strength and competitive anion. Desalin. Water Treat. 150, 274-281.

Lin, S.C., Gan, N., Qiao, L., Zhang, J.B., Cao, Y.T., Chen, Y.J., 2015. Magnetic metalorganic frameworks coated stir bar sorptive extraction coupled with GC-MS for determination of polychlorinated biphenyls in fish samples. Talanta 144, 1139-1145.

Liu, K., Zhang, S.Y., Hu, X.Y., Zhang, K.Y., Roy, A., Yu, G., 2015. Understanding the adsorption of PFOA on MIL-101(Cr)-based anionic-exchange metal-organic frameworks: comparing DFT calculations with aqueous sorption experiments. Environ. Sci. Technol. 49, 8657-8665.

Ma, J.P., Yao, Z.D., Hou, L.W., Lu, W.H., Yang, Q.P., Li, J.H., Chen, L.X., 2016. Metal organic frameworks (MOFs) for magnetic solid-phase extraction of pyrazole/pyrrole pesticides in environmental water samples followed by HPLC-DAD determination. Talanta 161, 686-692.

Ma, J.P., Wu, G.G., Li, S., Tan, W.Q., Wang, X.Y., Li, J.H., Chen, L.X., 2018. Magnetic 
solid-phase extraction of heterocyclic pesticides in environmental water samples using metal-organic frameworks coupled to high performance liquid chromatography determination. J. Chromatogr. A 1553, 57-66.

Ma, J.P., Li, S., Wu, G.G., Wang, S.S., Guo, X.T., Wang, L.Y., Wang, X.Y., Li, J.H., Chen, L.X., 2019a. Preparation of mixed-matrix membranes from metal organic framework (MIL-53) and poly(vinylidene fluoride) for use in determination of sulfonylurea herbicides in aqueous environments by high performance liquid chromatography. J. Colloid Interface Sci. 553, 834-844.

Ma, J.Q., Liu, L., Wang, X., Chen, L.Z., Lin, J.M., Zhao, R.S., 2019b. Development of dispersive solid-phase extraction with polyphenylene conjugated microporous polymers for sensitive determination of phenoxy carboxylic acids in environmental water samples. J. Hazard. Mater. 371, 433-439.

Makvandi, P., Ghaemy, M., Mohseni, M., 2016. Synthesis and characterization of photocurable bis-quaternary ammonium dimethacrylate with antimicrobial activity for dental restoration materials. Eur. Polym. J. 74, 81-90.

Massoudinejad, M., Ghaderpoori, M., Shahsavani, A., Amini, M.M., 2016. Adsorption of fluoride over a metal organic framework Uio-66 functionalized with amine groups and optimization with response surface methodology. J. Mol. Liq. 221, 279-286.

Mhaka, B., Cukrowska, E., Bui, B.T.S., Ramström, O., Haupt, K., Tutu, H., Chimuk, L., 2009. Selective extraction of triazine herbicides from food samples based on acombination of a liquid membrane and molecularly imprinted polymers. J. Chromatogr. A 1216, 6796-6801.

Mojtaba, S., Nazir, F., Meghdad, P., Kiomars, S., 2012. Simultaneous preconcentration and determination of 2,4-D, alachlor and atrazine in aqueous samples using dispersive liquid-liquid microextraction followed by high-performance liquid chromatography ultraviolet detection. J. Sep. Sci. 20, 2714-2718.

Molavi, H., Eskandari, A., Shojaei, A., Mousavi, S.A., 2018. Enhancing CO2/N2 adsorption selectivity via post-synthetic modification of NH2-UiO-66(Zr). Microporous Mesoporous Mater. 257, 193-201.

Mondal, S., Xu, J.Q., Chen, G.S., Huang, S.M., Huang, C.Y., Yin, L., Ouyang, G.F., 2019. Solid-phase microextraction of antibiotics from fish muscle by using MIL-101(Cr) $\mathrm{NH} 2$-polyacrylonitrile fiber and their identification by liquid chromatographytandem mass spectrometry. Anal. Chem. Acta 1047, 62-70.

Otalvaro, M.A., Avena, M., Brigante, M., 2019. Adsorption of organic pollutants by amine functionalized mesoporous silica in aqueous solution. Effects of $\mathrm{pH}$, ionic strength and some consequences of APTES stability. J. Environ. Chem. Eng. 7, 103325.

Peng, M.M., Han, Y.Q., Xia, H., Hu, X.Z., Zhou, Y.X., Peng, L.J., Peng, X.T., 2018. Rapid and sensitive detection of the phenoxy acid herbicides in environmental water samples by magnetic solid-phase extraction combined with liquid chromatography-tandem mass spectrometry. J. Sep. Sci. 41, 2221-2228.

Rapti, S., Pournara, A., Sarma, D., Papadas, I.T., Armatas, G.S., Hassan, Y.S., Alkordi, M.H., Kanatzidis, M.G., Manos, M.J., 2016. Rapid, green and inexpensive synthesis of high quality UiO-66 amino functionalized materials with exceptional capability for removal of hexavalent chromium from industrial waste. Inorg. Chem. Front. 3, 635-644.

Sanagi, M.M., Muhammad, S.S., Hussain, I., Ibrahim, W.A.W., Ali, I., 2015. Novel solidphase membrane tip extraction and gas chromatography with mass spectrometry methods for the rapid analysis of triazine herbicides in real waters. J. Sep. Sci. 38, 433-438.
Sarker, M., Song, J.Y., Jhung, S.H., 2018. Carboxylic-acid-functionalized UiO-66-NH2: a promising adsorbent for both aqueous- and non-aqueous-phase adsorptions. Chem. Eng. J. 331, 124-131.

Sierke, J., Ellis, A.V., 2019. Cross-linking of dehydrofluorinated PVDF membranes with thiol modified polyhedral oligomeric silsesquioxane (POSS) and pure water, flux analysis. J. Membr. Sci. 581, 362-372.

Slingsby, R.W., Pohl, C.A., 1988. Anion-exchange selectivity in latex-based columns for ion chromatography. J. Chromatogr. A 458, 241-253.

Suárez, R., Clavijo, S., González, A., Cerdà, V., 2018. Determination of herbicides in environmental water samples by simultaneous in-syringe magnetic stirring-assisted dispersive liquid-liquid microextraction and silylation followed by GC-MS. J. Sep. Sci. 41, 1096-1103.

Wang, C.H., Liu, X.L., Chen, J.P., Li, K., 2015. Superior removal of arsenic from water with zirconium metal organic framework UiO-66. Sci. Rep. 5, 16613-16623.

Wang, J.Q., Zhu, J., Si, L., Du, Q., Li, H.L., Bi, W.T., Chen, D.D.Y., 2017. High throughput screening of phenoxy carboxylic acids with dispersive solid phase extraction followed by direct analysis in real time mass spectrometry. Anal. Chim. Acta 996, 20-28.

Wu, M.X., Yang, Y.W., 2017. Metal-organic framework (MOF)-based drug/cargo delivery and cancer therapy. Adv. Mater. 29, 1606134.

Wu, J., Ee, K.H., Lee, H.K., 2005. Automated dynamic liquid-liquid-liquid microextraction followed by high-performance liquid chromatography-ultraviolet detection for the determination of phenoxy acid herbicides in environmental waters. J. Chromatogr. A 1082, 121-127.

Xiang, S., Zhou, W., Zhang, Z., Green, M.A., Liu, Y., Chen, B., 2010. Open metal sites within isostructural metal-organic frameworks for differential phenoxy carboxylic acids (PCAs) recognition of acetylene and extraordinarily high acetylene storage capacity at room temperature. Angew. Chem. Int. Ed. 49, 4615-4618.

Xu, X., Gao, B.Y., Tan, X., Zhang, X.X., Yue, D.T., Yue, Q.Y., 2013. Uptake of perchlorate from aqueous solutions by amine-crosslinked cotton stalk. Carbohydr. Polym. 98, $132-138$.

Xue, D.X., Wang, Q., Bai, J.F., 2019. Amide-functionalized metal-organic frameworks: syntheses, structures and improved gas storage and separation properties. Coord. Chem. Rev. 378, 2-16.

Yang, Z.B., Zhu, L., Chen, L., 2019. Selective adsorption and separation of dyes from aqueous solution by core-shell structured $\mathrm{NH}_{2}$-functionalized UiO-66 magnetic composites. J. Colloid Interface Sci. 539, 76-86.

Zhang, S.N., Han, P.P., Xia, Y., 2017. Facile extraction of azide in sartan drugs using magnetized anion-exchange metal-organic frameworks prior to ion chromatography. J. Chromatogr. A 1514, 29-35.

Zhang, H., Wu, X.Q., Yuan, Y.N., Han, D.D., Qiao, F.X., Yan, H.Y., 2018. An ionic liquid functionalized graphene adsorbent with multiple adsorption mechanisms for pipettetip solid-phase extraction of auxins in soybean sprouts. Food Chem. 265, 290-297.

Zhao, Q., Zhang, S.Y., Zhang, X.J., Lei, L., Ma, W., Ma, C.X., Song, L., Chen, J.W., Pan, B., Xing, B.S., 2017. Cation-pi interaction: a key force for sorption of fluoroquinolone antibiotics on pyrogenic carbonaceous materials. Environ. Sci. Technol. 51, 13659-13667.

Zhu, X., Zheng, H.Y., Wei, X.F., Lin, Z.Y., Guo, L.H., Qiu, B., Chen, G.N., 2013. Metal-organic framework (MOF): a novel sensing platform for biomolecules. Chem. Commun. 49, 1276-1278. 